\title{
A study on structure and functions of organs involved in the formation of Mongolian Khuumii sound
}

\begin{abstract}
Rentsendorj Tsagaankhuu ${ }^{1}$, Enebish Sundui ${ }^{1}$, Juramt Bold ${ }^{1}$, Uurtuya Shuumarjav ${ }^{3}$, Ganchimeg Palamdorj ${ }^{2}$ Byambasuren Luvsandagva ${ }^{4}$, Dorjsuren Tsagaankhuu ${ }^{2}$, Erdembileg Tsevegmid ${ }^{5}$ Amgalanbaatar Dorjkhuu ${ }^{1}$, Dagdanbazar Bodi ${ }^{6}$ and Nyamdorj Dagdanbazar ${ }^{1 *}$

${ }^{1}$ Department of Anatomy, Mongolian National University of Medical Sciences, Ulaanbaatar, Mongolia.

${ }^{2}$ Department of Otorhinolaryngology, Mongolian National University of Medical Sciences, Ulaanbaatar, Mongolia.

${ }^{3}$ Department of Path-Physiology, Mongolian National University of Medical Sciences, Ulaanbaatar, Mongolia.

${ }^{4}$ Department of Ear, Nose and Throat, First Central Hospital, Ulaanbaatar, Mongolia.

${ }^{5}$ Department of Radiology, Mongolian National University of Medical Sciences, Ulaanbaatar, Mongolia.

${ }^{6}$ Department of Morphology, Ach Medical university, Ulaanbaatar, Mongolia.
\end{abstract}

\begin{abstract}
Khuumii (throat or overtone singing) is a unique form of art derived from the nomadic population of Central Asia, which is a type of singing in which the singer manipulates the resonances (or formants) created as air travels from the lungs, past the vocal folds, and out of the lips to produce a melody. A total of 60 participants, aged 18-60 years (54 men and 6 women), were selected by nonrandom sampling method using cross-sectional study. X-ray, endoscopy, and sound research method were used in the study, and the composition of blood gas was analysed. X-ray examination determined the state during each different types of Khuumii; Shakhaa and Kharkhiraa. As the basic timbre of Shakhaa Khuumii went up progressively the larynx grew and the compression strength increased, while the basic timbre went down, and the larynx became lower. In the case of Kharkhiraa Khuumii, the larynx position was elevated to a relatively small extent compared to Shakhaa Khuumii and the distance between the sublingual bone and the larynx was large. The sublingual bone trunk lowered during Shakhaa Khuumii, while it was slightly elevated during Kharkhiraa Khuumii. The laryngeal endoscopy evaluated the movement of true and false vocal chords, glottal volume, movements of epiglottis and arytenoid cartilage, and mucosa. Furthermore, the sound frequency is 2-4 times higher than that of normal speech, and sound volume is 0.5-1 times higher. The blood gas composition test showed partial pressure ( $\mathrm{pO}_{2}$ ), and saturation of oxygen $\left(\mathrm{SaO}_{2}\right)$ decreased after performing Khuumii. In the case of Shakhaa and Kharkhiraa Khuumi, it is appropriate to divide Khuumii into two main types according to structural and functional changes in the organs involved.
\end{abstract}

Keywords: throat singing; true vocal fold; false vocal fold; voice source; vibration mode; phoniatry;

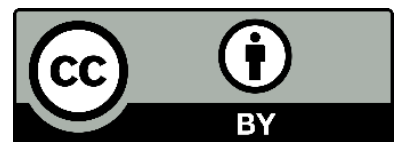

The Author(s). 2020 Open access This article is distributed under the terms of the Creative Commons Attribution 4.0 International License (https://creativecommons.org/licenses/by/4.0/), which permits unrestricted use, distribution, and reproduction in any medium, provided you give appropriate credit to the original author(s) and the source, provide a link to the Creative Commons license, and indicate if changes were made. 


\section{INTRODUCTION}

Khuumii is a unique form of art that originated from the nomadic people of Central Asia, producing two or more "simultaneous" sounds through the vocal chords [1]. The origin and development of Khuumii in Mongolia was confirmed by UNESCO and registered in the list of intangible cultural heritage of mankind in 2010 [2]. Scientists have determined that Khuumii as an art developed in some 15 countries around the world, and that it has developed from nomadic art to professional stage art in Mongolia [3] In recent years, the number of researchers studying Khuumii, in terms of medicine, is growing. A summary of literature review: In Mongolia, researcher BatOyun, Ch. "Some Issues of Mongolian Khuumii", Enkhjargal, Sh. "Features of Different Styles of Mongolian Khuumii", Mendbayar, J, "The Spread and Regional Features of Khuumii", Kherlen, L. "Mongolian Khuumii Meaning", Zagd-Ochir, S. "Some Issues about the Skills of Khuumii Performers", Tsogtgerel, Ts. "The Issues of Khuumii Methods", Odsuren, B. "Characteristics of Professional Training Methods of Mongolian Khuumii" [4-10]. Foreign researchers: Sakakibara K. (Japan) "Vocal fold and false vocal fold vibrations in throat singing and synthesis of khoomei”, Lindestad P. (Sweden) "Voice Source Characteristics in Mongolian 'Throat Singing' Studied with High-Speed Imaging Technique, Acoustic Spectra, and Inverse Filtering", Kharuto A. V. (Russia) "About The Musical And Acoustical Characteristics Of Tuva Throat Singing", Aulanko R. (Finland) "Acoustic Characteristics of Different Styles of Overtone Singing In Altai", Grawunder S. (Germany) "Comparison of voice production types of 'western' overtone singing and South Siberian throat singing”, Li G. (China) "The Physiological Basis of Chinese Нццтіi Generation" [11-16]. Researchers in our country have mostly studied the origin and development of Khuumii from the perspective of history, geography, ethnography, art, culture and linguistics, while foreign researchers have conducted studies in terms of phonology and physics. In any of these cases, the number of research subject is limited due to the small number of professional and amatuer Khuumii performing artists.

The lack of anatomical and physiological research and publications on Khuumii indicates the need for a scientific study on the Khuumii process, correction of some unscientific oral interpretations of Khuumii, and resolution of disputes over classification. Khuumii can be divided into many different types involving sound, timbre, organs, which are used when performing a Khuumii song based on Khuumii procedures and Khuumii singer's experiences. Some Khuumii singers and researchers have noted that Khuumii can be divided into two types - 1. Fluid Isgeree and 2. Kharkhiraa (D. Sundui), R. Davaajav had noted: there are 3 different types of Khuumii, including 1. Palate, 2. Pharynx and 3. Frequency, while T. Gandold has classified Khuumii as follows: 1 . Kharkhiraa, 2. Fluid Isgeree, 3. Outdoor 4. Nasal [17]. Therefore, the purpose of this study is to scientifically determine the structural and functional features of the organs involved in Khuumii using modern diagnostic equipment, methods and techniques. The study aimed to identify the anatomical structures involved in the formation of Khuumii sound and the features and patterns of their functions, and to compare each style of Khuumii.

The following objectives have been set in line with the aim of the study:

1. Identify anatomical structures that play a primary and auxiliary role in khuumii singing, as well as the functional features and correlation of these structures,

2. Propose classification of different styles of Khuumii from a medical point of view depending on the characteristics of structure and function described. 


\section{MATERIALS AND METHODS}

Cross-sectional study was conducted including a total of 60 participants aged 18-60 years (men; $n=54$, and women; $n=6$ ), selected by a non-random sampling method. The participants are professional and amateur Mongolian Khuumii performers at art organizations operating in different parts of Mongolia. Questionnaires and physical examinations (height, body weight, BMI) were administered in order to study some of the structural and functional parameters of the organs involved in producing Khuumii.

$\mathrm{X}$-ray examination was performed using Digital X-ray machine (Medien International Co. Ltd,,Korea), which recorded the image of Khuumii singing and the location, movement, and functional characteristics of the head and neck organs in producing Khuumii, and the images were studied.

Laryngoscopy was performed using $70^{\circ}$ KARL-STORZ straight endoscopes (Germany)

\section{RESULTS AND DISCUSSION}

90.7 per cent of the Khuumii performers were male and 9.3 per cent were female. The average height of the participants was $172.91 \pm 0.93 \mathrm{~cm}$ (arithmetic mean and mean error), average body weight was $77.53 \pm 2.46 \mathrm{~kg}$, and body mass index was $25.93 \pm 5.31$ respectively. Heart rate was $92.19 \pm 20.71$ per minute prior to Khuumii, which was $133.19 \pm 19.09$ bpm after performing Khuumii and $85.81-98.56 \mathrm{bpm}$ at 95 per cent confidence interval. 60.5 per cent of the participants were professional Khuumii singers who had graduated from relevant universities and colleges.

The questionnaire revealed whether there are changes in the function of the human vocal apparatus and the organs involved in sound production, depending on the type of Khuumii and prolonged Khuumii. The following results were obtained: 74.1 per cent of the participants did not have hoarse throat and 25.9 per cent had hoarse throat; 55.8 per cent of the participants experienced change in their tone and 44.2 per cent with no change in tone; 95.3 per cent did and, flexible endoscope (Japanese Fujifilm) to assess the movement of true and false vocal cords, glottal volume, movement of the epiglottis, arytenoid cartilage, and mucosa state.

Acoustic parameters such as sound frequency $\mathrm{Hz}$, sound power $\mathrm{Db}$, sound tone, amplitude, maximum sound output time, clear tone and noise ratio, and dynamic range were analysed by a sound analyser.

General blood test was performed using the OPTI CCA blood gas analyser (Sysmex XS1000i, USA), hematology analyser (Japan) investigating changes in the blood gas composition, $\mathrm{O}_{2}, \mathrm{CO}_{2}$ partial pressure, hemoglobin oxygen saturation, red blood cell and hemoglobin levels at normal state and after Khuumii, respectively. Statistical analysis was performed using SPSS-23 and Microsoft Excel softwares using basic biostatistical methods.

not have sore throat and 4.7 per cent had sore throat; 74.4 per cent did not experience strained voice and 25.6 per cent did; 88.4 per cent had no shortness of breath and 11.6 per cent had shortness of breath; 81.4 per cent had no palpitation and 18.6 per cent had; 53.5 per cent do not consume alcohol at all and 39.5 per cent consume a little; and 81.4 per cent did not have Khuumii performers in their family.

Results of X-ray analysis:

$X$-ray examination determined the state during each of the two different types of Khuumii - Shakhaa and Kharkhiraa. During Shakhaa Khuumii from normal state, the larynx is raised, joining the sublingual bone and pressed up to the chin. Sublingual bone is lowered by trunk tilted to 45 degrees, epiglottis cartilage is bent forward to tongue root and its position changed according to the movement of the tongue, the soft palate is lifted up, and the nasopharynx is completely separated and closed from the mouth. As the basic timbre of Shakhaa Khuumii went up, the larynx raised up and the compression strength increased, 
whereas the base note went down, and the larynx lowered. Sound of Khuumii coming from the larynx resonated through the lower and middle parts of the pharynx and further through the mouth cavity (Figure 1).

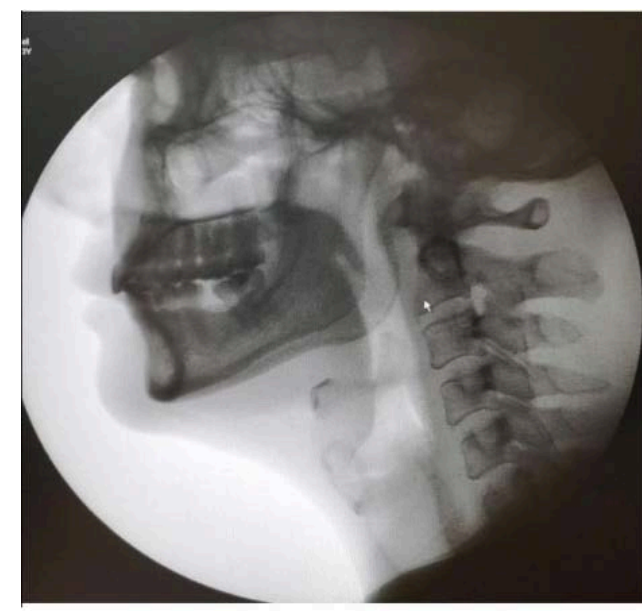

Quiet breathing

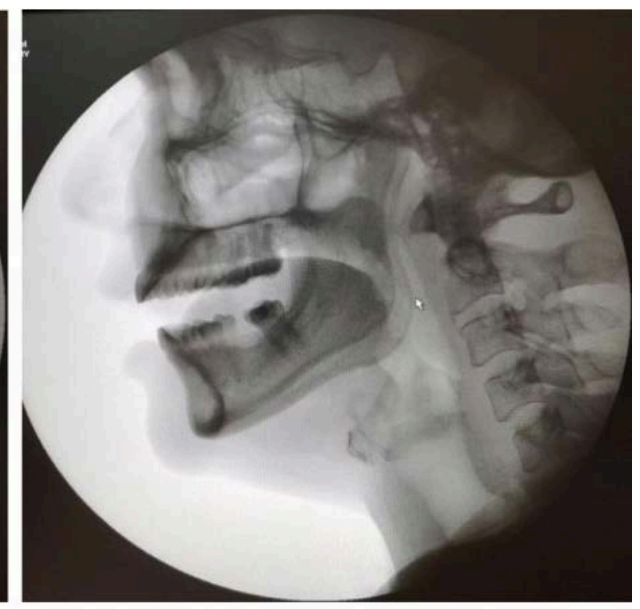

Singing "Shakhaa" Khuumii

Figure 1. This figure illustrates the location of the organs during Shakhaa Khuumii singing

During Isgeree Khuumii, in addition to the basic changes that occur in Shakhaa Khuumii, the position of the tongue is elevated and touches the hard palate making fine movements, basic tone of Shakhaa Khuumii coming from the larynx is shaped by changes in the position and movement of the tongue creating clear and soft Isgeree (whistling) sound. As the tone of Isgeree goes up, the position of the tongue shifts to the hard palate and upper anterior teeth, and as the tone comes down, the tongue weakens and moves backwards (Figure 2).

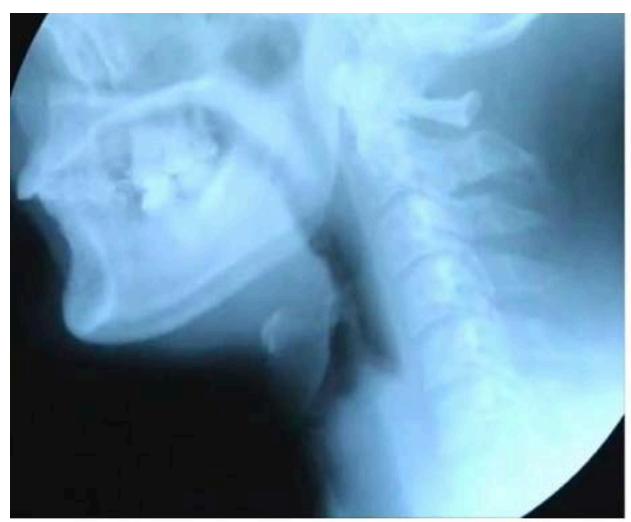

Quiet breathing

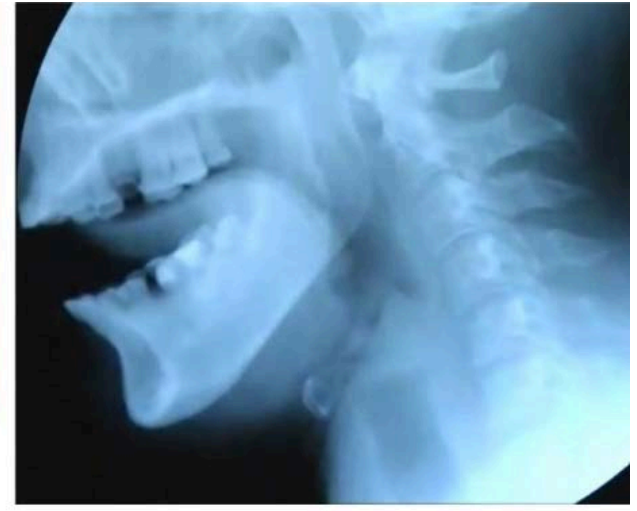

Singing "Isgeree" Khuumii

Figure 2. The location of the organs during Isgeree Khuumii singing

In the case of Kharkhiraa Khuumii, the larynx position was elevated to a relatively small extent compared to Shakhaa Khuumii and the distance between the sublingual bone and the larynx was large. The sublingual bone trunk lowered during Shakhaa Khuumii, while it was slightly elevated during Kharkhiraa Khuumii.
As the tone of Kharkhiraa Khuumii goes up and down, the position of the larynx changes in direct proportion. X-rays showed that the soft palate was completely closed, the vocal cords made wave-like movements, and the frequency changed as the sound changed tone (Figure 3 ). 


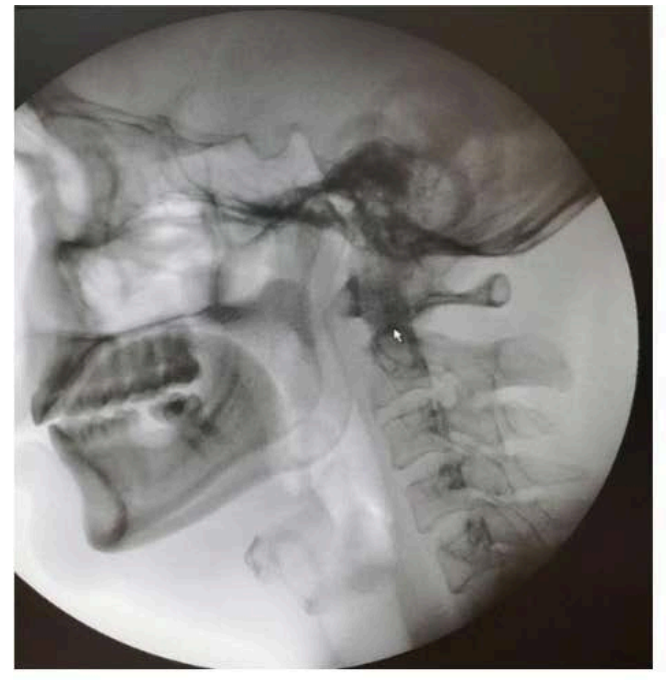

Quiet breathing

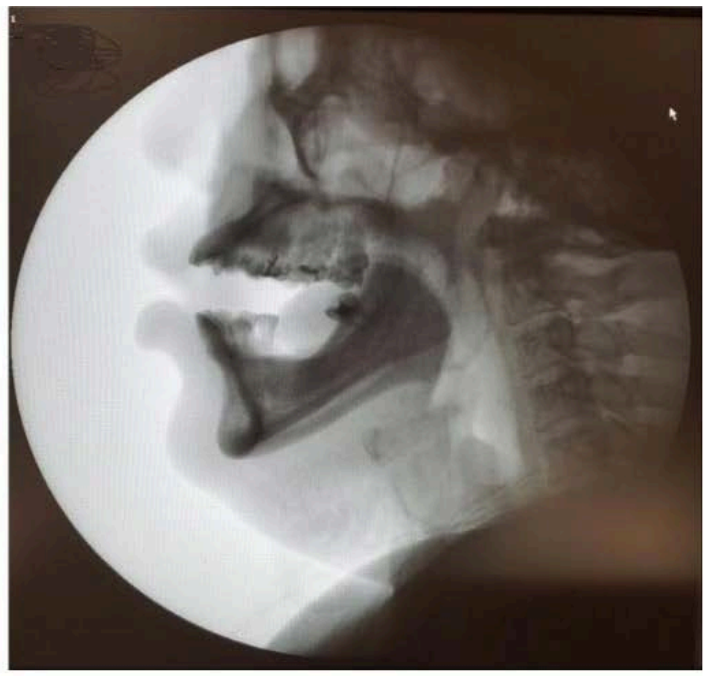

Singing "Kharkhiraa" Khuumii

Figure 3. The location of the organs during Kharkhiraa Khuumii on X-ray

\section{Results of laryngoscopy:}

Shakhaa (Isgeree) khuumii: During Khuumii singing, the false vocal cords were closed on all sides. The glottis narrows and becomes a hole. Isgeree Khuumii, on the other hand, produces a subtle whistling sound when the tongue touches the palate on top of Shakhaa
Khuumii method. Therefore, the position and movement of the larynx are similar to that of changes in Shakhaa Khuumii, but as the movement of the tongue is involved, the movement of arytenoid cartilage is increased by the movement of the tongue.
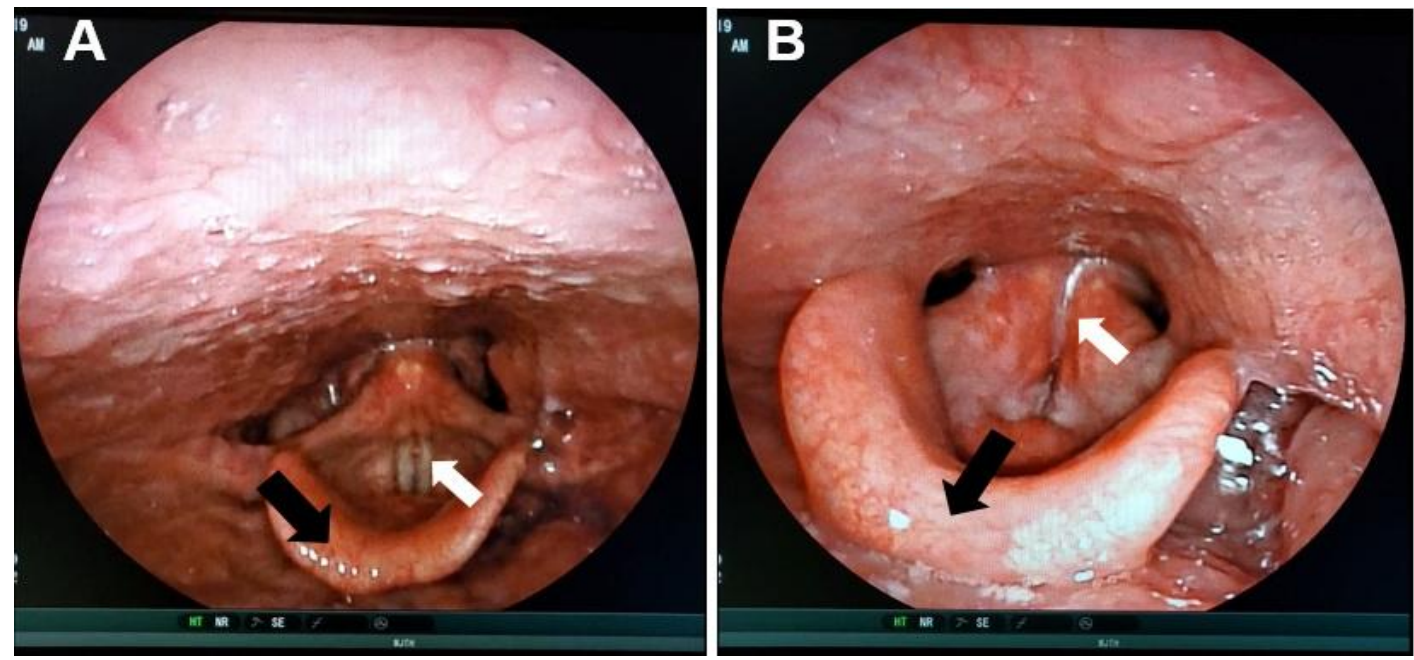

Figure 4. The anatomical structure of the vocal folds during Shakhaa Khuumii singing (laryngoscopy) A. Normal structure of larynx, B. Structure of larynx during Shakhaa Khuumii singing

Isgeree Khuumii is required to be forced with a higher tone to produce a subtle tone, so the gap between the glottis was compressed more and more. In other words, it was observed that a single Khuumii tone is formed by vibrations in the middle third of the laryngeal vestibule or between the mucous membranes of the vocal folds (Figure 4, Figure 5). 


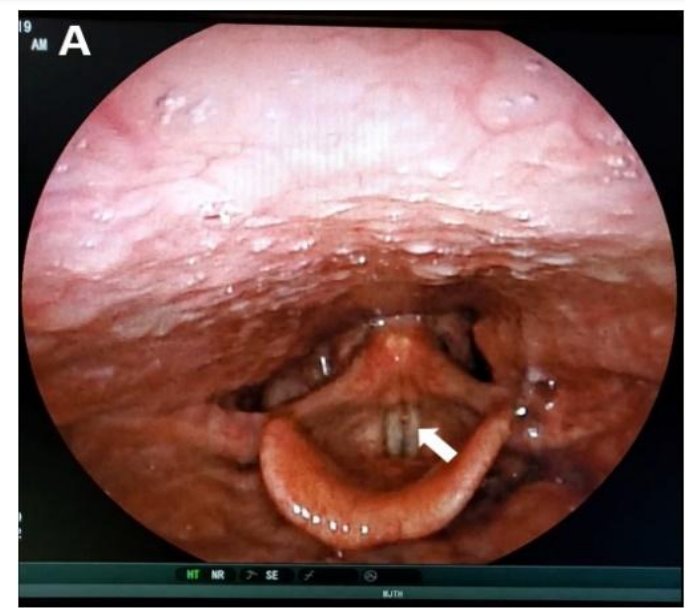

Figure 5. The anatomical structure of the vocal folds during Isgeree Khuumii singing (laryngoscopy) A. Normal structure of larynx, B. Structure of larynx during Isgeree Khuumii

Kharkhiraa Khuumii: Some researchers have noted that Kharkhiraa Khuumii is similar to the sounds of lions roaring and wolves howling. Linguistically, the word Kharkhiraa means "hoarse and thick voice" and vowels such as "A", "U", and "O" are predominant in the Khuumii. The contraction force of the false vocal cords is relatively weak compared to that of the Shakhaa Khuumii, and glottis is slightly visible. The vocal cords are intermittently contracted and sound is heard as vibration during Kharkhiraa Khuumii. In other words, at this time, creating vibrations in the anterior third of the laryngeal vestibule or between the mucous membranes of the vocal folds were observed to form Kharkhiraa Khuumii sound. The mucous membrane of the posterior third of the laryngeal vestibule can also vibrate, and if these structures vibrate at once, simultaneous sound and melody of Khuumii is produced. (Figure 6).
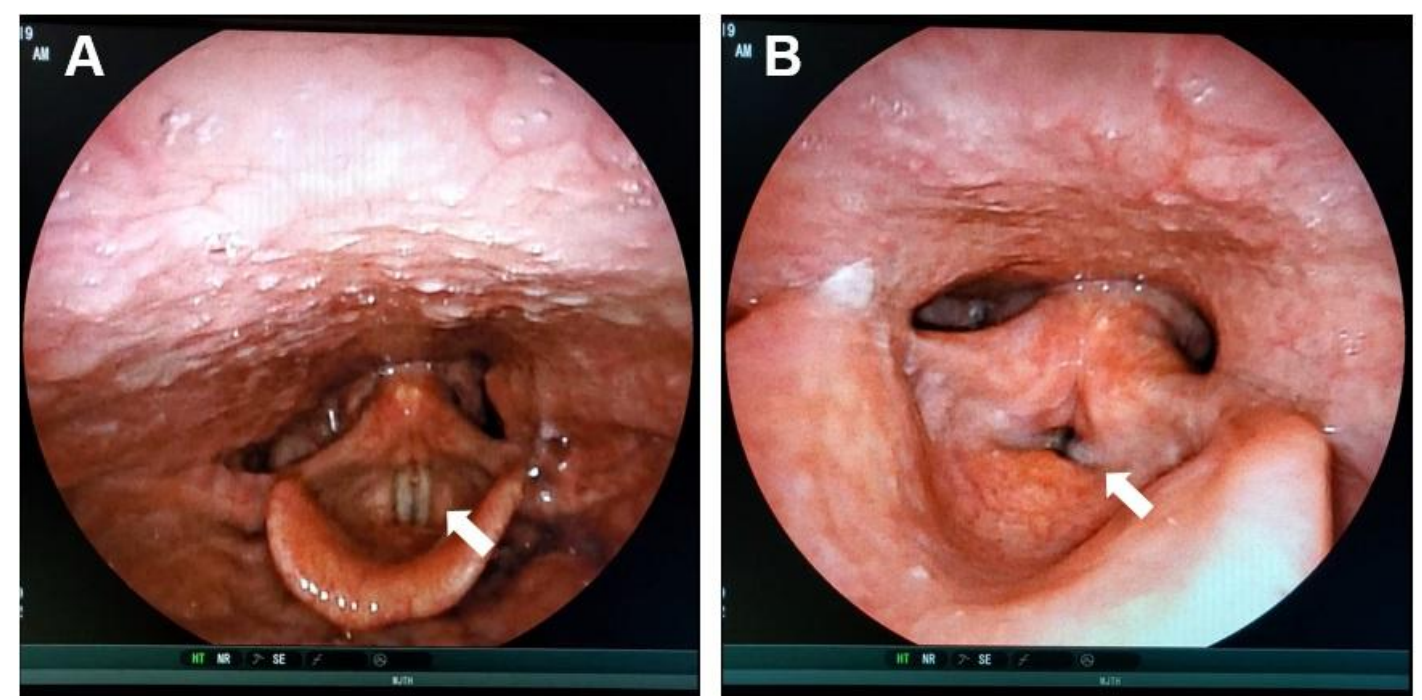

Figure 6. The anatomical structure of the vocal folds during Kharkhiraa Khuumii singing (laryngoscopy) A. Normal structure of larynx, B. Structure of larynx during Kharkhiraa Khuumii

\section{Results of study on changes in blood gas composition during Khuumii singing:}

After 10 minutes of Khuumii by the participants, 75 per cent was alkaline, and the partial pressure of oxygen $\left(\mathrm{pO}_{2}\right)$ had decreased by 90 per cent compared to normal range. The partial pressure of carbon dioxide $\left(\mathrm{pCO}_{2}\right)$ was 35 per cent hypocapnia, 5 per cent hypercapnia in all participants. Saturation of oxygen $\left(\mathrm{SaO}_{2}\right)$ was reduced in all participants. The averaged range of $\mathrm{pO}_{2}$ was $47 \mathrm{mmHg}$ and the average range of $\mathrm{SaO}_{2}$ was 82 per cent. 
$\mathrm{SaO}_{2}$ was 91 per cent before Khuumii and dropped to 82 per cent after 10 minutes of performing Khuumii. In the blood gas analysis of all participants, the average range of partial pressure of oxygen gas was $57.25 \mathrm{mmHg}$ and decreased to $47 \mathrm{mmHg}$ after 10 minutes of Khuumii. During Khuumii, the $\mathrm{pO} 2$ and $\mathrm{SaO} 2$ are reduced.

\section{Results of study on some acoustic characteristics of sound during Khuumii}

The study was done to determine some acoustic indicators of the two main styles of Khuumii - Shakhaa, and Kharkhiraa: the average sound of frequency was $795 \pm 15 \mathrm{~Hz}$ for Shakhaa Khuumii, and $120 \pm 10 \mathrm{~Hz}$ for Kharkhiraa Khuumii, which are 2-4 times higher as compared to the frequency of ordinary speech. The sound power of Shakhaa Khuumii was $95.0 \pm 5.0 \mathrm{Db}$, and $82.0 \pm 5.0 \mathrm{Db}$ for Kharkhiraa Khuumii, which are 7-10 times stronger than ordinary speech. The objective of the study was to examine the structural and functional features of some of the organs involved in khuumii. According to the results of the questionnaire, 60.5 per cent of the participants were professional Khuumii singers with university degrees, which indicates that the recent enrolments in Khuumii major is related to the educational system and training. 9.3 per cent were self-learners of Khuumii, which indicates that Khuumii skills exist in the Mongolian gene pool.

One of the changes in the body during Khuumii (throat singing) was an increase in the number of heartbeats. We have tried to explain how the force formed from Khuumii affects the human body. The pressure on the body is calculated by maximum heart rate (MHR) using a special formula. This formula is based on the calculation of the heart rate as a percentage of the difference between the number of heartbeats at rest and after Khuumii and classified as follows: 50-60 per cent as low, 60-70 per cent as medium, 70-80 per cent as quite demanding of muscle strength, and 100 per cent has been classified as the maximum strength required. MHR after Khuumii reaches 70-80 per cent or requires muscle strength. Considering theoretically from the above explanation and the state of physical strain, following can be adduced: Increased cardiac activity during
Khuumii can be explained by the activation of the sympathoadrenal system and the secretion of catecholamines. 81.4 per cent of the participants did not experience an increase in heart rate, indicating that the cardiovascular function is normal and adaptable. However, 18.6 per cent reported an increase in heartbeats, which may be explained by an increase in the pressure on the heart during Khuumii and a lower ability to adapt to the load. Therefore, Khuumii singers need to be under medical supervision of a cardiologist. Furthermore, 53.5 per cent of the participants smoke, which indicates a high risk of respiratory disease, which expresses the need to completely give up smoking, a factor that affects the healthy voice. Another part of the questionnaire was the result clarified whether changes in the function of the human vocal apparatus and the organs involved in sound production depend on the duration of Khuumii and prolonged Khuumii. When performing Khuumii in short and long versions, mucous membranes of the laryngeal vocal cord and the laryngeal vestibule ligament are determined to be not swollen. 27.9 per cent said that they had hoarseness in their throat, suggesting new learners or hoarseness for other reasons. 55.8 per cent of the participants answered that their voice tone changed, which is likely due to physiological enlargement that may lead to functional changes in vocal apparatus, as well as may depend on the duration of Khuumii singing. However, 44.2 per cent of the questioned answered that there is no change in their voice tone, which may be related to proper technique of Khuumii and genetics. 95.3 per cent of the participants did not experience sore throat, indicating that the pharyngeal and laryngeal mucous membranes do not damage from Khuumii. It is reasonable to assume that 4.7 per cent had sore throat due to reasons other than Khuumii. 97.7 per cent replied they did not have any pain when swallowing, which suggests that the pharyngeal mucous membranes do not irritate and are not damaged by Khuumii. 74.4 per cent of the participants said that their voice did not strain, presenting that if learned correctly, vocal apparatus muscles do not strain. Whereas, 25.6 per cent of the participants said that they experience strained voice, which is related to 
their learning technique. Further, a detailed study in relation to the duration (years) of performing Khuumii is required. There are two theories that explain Khuumii: the "doublesource" theory and the "resonance theory". Chernov and Maslov's (1989) "double-source" theory proposes that along with the vibration of the vocal cords, a narrow primary pitch similar to whistling is produced at the rear of the vocal cords [18]. However, Bloothoof's (1992) "resonance theory" suggests that the primary pitch is formed from oscillations of the vocal cords, melody resonates and that the narrow high note is heard separately from the other components of the sound. The study observed Tuva's ethnic Sygyt throat singing through inserting flexible laryngoscopy into the nasal cavity and found that both true and false vocal cords were tightly closed, creating a whistling sound with a narrow gap in the rear. Furthermore, function of the pharynx, larynx, and arytenoid cartilage were examined every time the sound changed. Stroboscopic examination of the throat with a direct laryngoscopy pulling the Khuumii performer's tongue revealed a continuous Khuumii sound without a melody [19].

\section{CONCLUSIONS}

During the formation of Khuumii sound, thoracic cavity, diaphragm, and lungs regulate the intensity of the air reaching the vocal folds, exert pressure on the airways and vibrate the sound waves through air flows, passing through the larynx and vocal folds. Mouth-nose cavity
This was similar to the process of Shakhaa Khuumii in our study, but the fact that the lower part of the tongue and pharynx play an important role in the formation of Shakhaa khuumii shows that it is similar to the Isgeree style of Mongolian Khuumii. It also proves that the melody of Khuumii is formed by a mechanism or by the involvement of other resonating structures, rather than the double pitch produced by the larynx. Adachi and Yamada (1999) concluded that MRI images of Khuumii shows that the tongue is raised to form a melody [20]. These researchers also concluded that the true vocal cords come in close contact and joins with false vocal cords to form basic note, and further primary pitch resonates when the tongue is lifted, which is unquestionably in line with the results of X-ray and laryngoscopy of our research. Klingholz (1993) determined that the basic sound frequency was $202 \mathrm{~Hz}$ for Shakhaa (Sygyt) Khuumii and $100-200 \mathrm{~Hz}$ for kargyraa (Kharkhiraa), which is close to the result of acoustic parameters of Isgeree and Kharkhiraa Khuumii in our research [21].

as well as pharynx are responsible for resonating the sound.

It is proposed to classify Khuumii into two main classifications of Shakhaa and Kharkhiraa according to the structural and functional changes in the organs involved in Khuumii.

\section{REFERENCES}

1. Behar, A., Kumar, M., Kunov, H., A look at throat singing. Can Acoust - Acoust Can. 2001, 29(1).

2. Enkhbat G. Training on the implementation of the Convention for the Safeguarding of the Intangible Cultural Heritage, the role and capacity of the media. In: Darkhan-Uul aimag, Mongolia: Soyombo printing, 2017, p. 17.

3. Government of Mongolia. "Mongol Khöömii” program. Mongolia, 2007.
4. Bat-Oyun Ch. "Some issues of Mongolian throat singing” monograph, Ulaanbaatar, 2002.

5. Enkhjargal Sh. "Features of Mongolian khuumii species”, monograph, Ulaanbaatar, 2006.

6. Mendbayar J. "Features of Khöömiin distribution region” monograph, Ulaanbaatar, 2009.

7. Kherlen L. The meaning of Mongolian Höömei. Ulaanbaatar, 2010. 
8. Zagd-Ochir S. "Some issues of Mongolian throat singers skills" monograph, Ulaanbaatar, 2012.

9. Tsogtgerel Ts. "Issues of throat singing technique” monograph, Ulaanbaatar, 2013.

10. Odsuren B. "Features of Mongolian throat singing professional academic methods" monograph, Ulaanbaatar, 2014.

11. Sakakibara K., Imagawa H., Konishi T. et al. Vocal fold and false vocal fold vibrations in throat singing and synthesis of khöömei. Proc IntComput Music Conf Havana, Cuba. 2001, pp. 135-138.

12. Lindestad P. Å. , Södersten M., Merker B., Granqvist S. Voice source characteristics in Mongolian "throat singing" studied with high-speed imaging technique, acoustic spectra, and inverse filtering. J Voice. 2001,15(1): pp. 78-85. doi:10.1016/S08921997(01) 00008-X.

13. Kharuto A V. XX Session of the Russian Acoustical Society Moscow, October 2731, 2008. Water. 2008, 1, pp. 391-394.

14. Aulanko R., Harvilahti L. Acoustic characteristics of different styles of overtone singing in Altai, Reijo Aulanko and Lauri Harvilahti. :985-988.
15. Grawunder S., Comparison of voice production types of "western" overtone singing and South Siberian throat singing. 15th ICPhS Proc. 2003:1699-1702.

16. Nyamdorj D., Enenbish S., Rentsendorj Ts., Juramt B. et al., "Монгол Хөөмий Судлал” 2020 (in Mongolian)

17. Li G, Hou Q. The Physiological Basis of Chinese Höömii Generation. J Voice. 2017;31(1):116.e13-116.e16. doi:10.1016/ j.jvoice. 2016.03.007

18. Chernov B., Maslov V., 1989 "Larynx double sound generator" Proc. XI Congress of Phonetic Sciences 6, pp. 4043.

19. Bloothoof G., Bringmann E., Cappellen M., 1992 "Acoustics and perception of overtone singing” Journal of Acoustical Acoustical Society America 92(4), 101827-101827.

20. Adachi S., Yamada M., 1999. "An acoustical study of sound production in biphonic singing." Journal of Acoustic Society America 105(5), pp. 2920-2932.

21. Klingholz F., 1993 "Overtone singing: Productive mechanisms and Acoustic data” Journal of Voice 7(2), pp. 118-122. 